\title{
Ocorrência de parasitos gastrointestinais e fatores de risco de parasitismo em gatos domésticos urbanos de Santa Maria, RS, Brasil
}

\author{
Occurrence of gastrointestinal parasites and parasitism risk factors in domestic cats in Santa \\ Maria, RS, Brazil
}

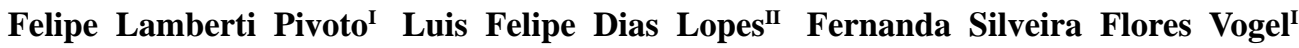 \\ Sônia de Avila Botton ${ }^{I}$ Luis Antonio Sangioni ${ }^{{ }^{*}}$
}

\section{RESUMO}

Neste trabalho, verificou-se a ocorrência de parasitos gastrointestinais em gatos urbanos domiciliados no município de Santa Maria, RS, no período de março a junho de 2011. Além disso, foram avaliados os fatores de riscos relacionados com a infecção dos parasitos gastrointestinais. Coletou-se 191 amostras de fezes de felinos para a realização de exames coproparasitológico e aplicado um questionário epidemiológico aos proprietários, contendo questões relativas aos hábitos e manejo dos seus animais. Das amostras fecais analisadas, 47,1\% (90/191) foram positivas para um ou mais parasitas gastrointestinais, sendo $33,5 \%$ (64/191) identificadas como mono-infecção e 13,6\% (26/191) como multi-infecção. Toxocara spp. (18,8\%) e Giardia spp. $(4,2 \%)$ foram os principais parasitos encontrados na mono-infecção. Na multi-infecção, as principais associações de parasitos detectadas incluíram: Ancylostoma spp. + Toxocara spp. $(3,1 \%)$ e Cystoisospora spp. + Cryptosporidium spp. + Giardia spp. + Toxoplasma spp. + Toxocara spp. (1,5\%). Os dados epidemiológicos foram submetidos ao teste qui-quadrado, Fischer, análise da relação de chances (OR), também analisada a relação entre os diferentes fatores epidemiológicos e a presença de parasitos gastrointestinais $(P>0,05)$. Os principais fatores de risco identificados foram: a escolaridade dos proprietários e a frequência de realização de tratamentos antiparasitários, os quais exerceram influência direta na ocorrência de parasitos gastrointestinais. Os dados obtidos contribuem para o conhecimento da epidemiologia das parasitoses gastrointestinais, bem como poderão auxiliar médicos veterinários e proprietários na elaboração de estratégias de controle e profilaxia antiparasitária para gatos domésticos.

Palavras-chave: endoparasitas, epidemiologia, Felis catus domesticus, sanidade.

\section{ABSTRACT}

This study verified the gastrointestinal parasites occurrence in household cats in Santa Maria, RS, Brasil from
March to June, 2011. In addition, the risk factors associated with gastrointestinal parasites infection were evaluated. One hundred ninety -one felines fecal samples were submitted to parasitological examination, as well as to an epidemiological questionnaire about the management and the animal habits, which was applied to the owners. Fecal samples were positive for one or more gastrointestinal parasites $(47.1 \%$ - 90/191). Occurrence of mono-infection was $33.5 \%$ (64/191) and multi-infection $13.6 \%$ (26/191). Toxocara spp. (18.8\%) and Giardia spp. (4.2\%) were the main parasites found in mono-infection. The main association of parasites detected included: Ancylostoma spp. + Toxocara spp. (3.1\%) and Cystoisospora spp. + Cryptosporidium spp. + Giardia spp. + Toxoplasma spp. + Toxocara spp. $(1.5 \%)$. Epidemiological data were submitted to chi-square, Fisher and odds ratio tests, as well as analyzed the relationship between the epidemiological factors and the presence of gastrointestinal parasites $(P>0.05)$. The main risk factors identified were: the scholar level of owners and frequency of antiparasitic treatments, which showed a direct influence on the occurrence of gastrointestinal parasites. The data contribute to the understanding of the epidemiology of gastrointestinal parasites and could aid veterinarians and owners in developing of antiparasitic strategies for domestic cats.

Key words: endoparasites, epidemiology, risk, Felis catus domesticus, health.

\section{INTRODUÇÃO}

Os estudos sobre o parasitismo em animais de estimação vêm despertando crescente interesse, devido à relação de proximidade existente entre o homem e os animais. Esse fato implica maiores cuidados à sanidade desses animais, visando a diminuir os riscos de transmissão de doença ao

\footnotetext{
IDepartamento de Medicina Veterinária Preventiva, Centro de Ciências Rurais (CCR), Universidade Federal de Santa Maria (UFSM), Avenida Roraima, 1000, prédio 44, sala 5149, Campus Universitário, Camobi, 97105-900, Santa Maria, RS, Brasil. E-mail: lasangioni@ gmail.com.

*Autor para correspondência.

IDepartamento de Ciências Administrativas, Centro de Ciências Sociais e Humanas (CCSH),UFSM, Santa Maria, RS, Brasil. Recebido 17.01.13 Aprovado 24.02.13 Devolvido pelo autor 07.06.13
} 
homem. No entanto, essas parasitoses podem desencadear diferentes agravos à saúde humana e animal, tais como: alergias, diarreias, anemias, entre outros (ALMEIDA \& AYRES, 2006).

Os gatos domésticos são considerados importantes reservatórios de endoparasitos, os quais acabam contaminando locais públicos, especialmente aqueles frequentados por crianças, expondo outros animais domésticos e o homem a um maior risco de infecção. Diversos aspectos epidemiológicos podem influenciar a ocorrência dos parasitos gastrointestinais em felinos domésticos, tais como: as medidas higiênico-sanitárias adotadas pelos cuidadores, o grau de escolaridade dos proprietários, a idade dos animais, o sexo, a raça, o status imunológico do hospedeiro, a condição corporal dos animais, os números de tratamentos antiparasitários realizados, dentre outros (BALASSIANO et al., 2009).

A finalidade deste estudo foi avaliar a ocorrência e os fatores de risco dos principais parasitos gastrointestinais que acometem os gatos domésticos urbanos de Santa Maria, RS.

\section{MATERIAIS E MÉTODOS}

Área do estudo e amostras

Foram realizadas visitas aleatórias em residências e criatórios de gatos urbanos do município de Santa Maria, Rio Grande do Sul, durante o período de março a junho de 2011, de acordo com o termo de consentimento dos proprietários. $\mathrm{O}$ cálculo amostral foi baseado em populações infinitas, segundo THURSFIELD (2004), totalizando 191 amostras fecais de gatos domésticos. As amostras fecais coletadas foram devidamente identificadas, acondicionadas em sacos plásticos e posteriormente em caixas térmicas e transportadas até o Laboratório de Doenças Parasitárias do Departamento de Medicina Veterinária Preventiva da Universidade Federal de Santa Maria, sendo realizados os exames coproparasitológicos até 72 horas após a coleta.

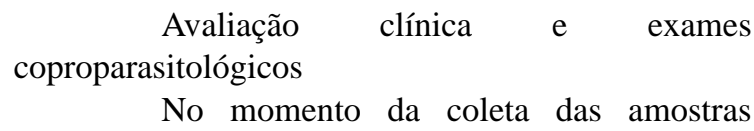
fecais, realizou-se o exame clínico dos gatos, avaliando os parâmetros vitais e o estado de saúde de todos os animais. Para a realização dos exames coproparasitológicos, foram empregadas as técnicas de Sedimentação Simples, Willis-Mollay, 1921 Modificada e Faust et al., 1938 Modificada, conforme descritas por MONTEIRO (2010).
Questionário epidemiológico

Um questionário epidemiológico foi aplicado aos proprietários dos animais no momento da coleta das amostras fecais. Esse questionário continha questões relativas à: raça (sem raça definida ou raça pura); sexo (macho ou fêmea); idade do animal (até 60 dias, de 2 a 6 meses, 6 meses a 1 ano, 1 a 5 anos, mais de 5 anos); tipo de alimentação ofertada (ração ou comida caseira); tipo de alojamento (casa, apartamento, centro de criação); acesso à área pública (sim ou não); frequência de tratamento antiparasitário (1/ano, 2/ ano, 3/ano, +3/ano, não tratado); higienização do meio ambiente (sim ou não) e escolaridade do proprietário (ensino fundamental, médio ou superior).

\section{Análise estatística}

Os resultados encontrados foram submetidos ao teste qui-quadrado, com auxílio do programa SAS (Statistical Analisys System) versão 9,2 , no qual foi analisada a relação entre os diferentes fatores epidemiológicos e a presença de parasitos gastrointestinais, também foi realizado o teste Fisher e análise da relação de chances (odds ratio, OR), com o auxílio do programa Graphpad Prism, considerando um nível de significância de $95 \%$.

\section{RESULTADOS E DISCUSSÃO}

Das 191 amostras fecais analisadas, 47,1\% (90/191) foram positivas para um ou mais parasitas gastrointestinais. A ocorrência encontrada neste estudo é compatível com os valores obtidos em diferentes regiões do Brasil, os quais encontraram prevalências que variaram de $31,5 \%$ a $100 \%$ (SERRA et al., 2003; FUNADA et al., 2007; COELHO et al., 2009; STALLIVIERE et al., 2009; DALL'AGNOL et al., 2010). Nas amostras analisadas, 33,5\% (64/191) foram identificadas como mono-infecção e em 13,6\% (26/191) como multi-infecção. Toxocara spp. e Giardia spp. foram os principais parasitos encontrados na mono-infecção, com ocorrência de $18,8 \%$ e $4,2 \%$, respectivamente. Entretanto, na multi-infecção, as principais associações de parasitos detectadas incluíram: Ancylostoma spp. + Toxocara spp. e Cystoisospora spp. + Cryptosporidium spp. + Giardia spp. + Toxoplasma spp. + Toxocara spp., com ocorrência de $3,1 \%$ e $1,5 \%$, respectivamente (Tabela 1).

PALMER et al. (2008) obtiveram 15,7\% das amostras avaliadas com mono-infecção, enquanto que a multi-infecção estava presente em apenas $2,7 \%$ dos animais estudados. No entanto, na Romênia, MIRCEAN et al. (2010) obtiveram uma prevalência 
Tabela 1 - Tipo de infecção e ocorrência de parasitos gastrointestinais em gatos domésticos de Santa Maria, RS, realizadas no período de março a junho de 2011.

\begin{tabular}{|c|c|}
\hline Mono infecção & Ocorrência (\%)(positivo/infectados) \\
\hline Toxocara spp. & $18,8(36 / 191)$ \\
\hline Giardia spp. & $4,2(08 / 191)$ \\
\hline Cystoisospora spp. & $3,1(06 / 191)$ \\
\hline Cryptosporidium spp. & $3,1(06 / 191)$ \\
\hline Ancylostoma spp. & $2,6(05 / 191)$ \\
\hline Paragonimus spp. & $0,5(01 / 191)$ \\
\hline Spirometra spp. & $0,5(01 / 191)$ \\
\hline Capillaria spp. & $0,5(01 / 191)$ \\
\hline Total & $33,5(64 / 191)$ \\
\hline \multicolumn{2}{|l|}{ Multi-infecção } \\
\hline Ancylostoma spp. + Toxocara spp. & $3,1(06 / 191)$ \\
\hline Cystoisospora spp. + Toxocara spp. & $1,0(02 / 191)$ \\
\hline Cryptosporidium spp. + Toxocara spp. & $0,5(01 / 191)$ \\
\hline Toxocara spp. + Toxascaris spp. & $0,5(01 / 191)$ \\
\hline Cryptosporidium spp. + Spirometra spp. & $0,5(01 / 191)$ \\
\hline Giardia spp. + Spirometra spp. & $0,5(01 / 191)$ \\
\hline Paragonimus spp. + Toxocara spp. & $0,5(01 / 191)$ \\
\hline Cryptosporidium spp. + Giardia spp. + Toxoplasma spp. & $0,5(01 / 191)$ \\
\hline Cystoisospora spp. + Entamoeba spp. + Sarcocystis spp. & $0,5(01 / 191)$ \\
\hline Cystoisospora spp. + Paragonimus spp. + Toxocara spp. & $0,5(01 / 191)$ \\
\hline Cystoisospora spp. + Sarcocystis spp. + Toxocara spp. & $0,5(01 / 191)$ \\
\hline Cystoisospora spp. + Cryptosporidium spp. + Toxocara spp. & $1,0(02 / 191)$ \\
\hline Cystoisospora spp. + Cryptosporidium spp. + Giardia spp. + Toxocara spp. & $1,5(03 / 191)$ \\
\hline Cystoisospora spp. + Cryptosporidium spp. + Sarcocystis spp. + Toxocara spp. & $0,5(01 / 191)$ \\
\hline Cystoisospora spp. + Cryptosporidium spp. + Giardia spp. + Toxocara spp. + Toxoplasma spp. & $1,5(03 / 191)$ \\
\hline Total & $13,6(26 / 191)$ \\
\hline
\end{tabular}

de animais com multi-infecção em 17,6\%, similar aos resultados evidenciados neste estudo. Todavia, no trabalho realizado por COELHO et al. (2009), no município de Andradina - SP, observou-se uma prevalência de multi-infecção em 76,4\% das amostras analisadas, diferindo dos resultados encontrados nesta pesquisa. As variações nos resultados observados podem ser explicadas, em parte, pela sensibilidade das técnicas laboratoriais empregadas nos diagnósticos dos parasitos, assim como devido à contaminação de formas infectantes dispersas no meio ambiente e as características geográficas, especialmente a temperatura, a umidade e a incidência solar, que influenciam na epidemiologia dos parasitos.

Toxocara spp. e Giardia spp. foram os parasitos mais observados nas amostras analisadas (Tabela 1). Em estudos anteriores, utilizando técnicas coproparasitológicas de centrifugo flutuação em gatos domiciliados, FUNADA et al. (2007) encontraram $6,1 \%$ de animais positivos para Toxocara cati e 2,1\% para Ancylostoma spp. Entretanto, SERRA et al. (2003), no Rio de Janeiro - RJ, verificaram, por meio de exames coproparasitológicos de 65 gatos domiciliados, a prevalência de $26,1 \%$ para Ancylostoma spp. e 9,2\% para Toxocara spp. No estudo de LORENZINI et al. (2007), realizado em fezes de gatos domiciliados na cidade de Porto Alegre -RS, foi constatada a prevalência para Toxocara spp. de 5,9\% e para Ancylostoma spp. de 3,8\%. Todos esses relatos comprovam a presença de parasitos causadores de zoonose em gatos domésticos. Esses dados salientam a importância da realização de tratamento antiparasitário adequado nos animais de estimação, a fim de reduzir o risco de infecção do homem pelos parasitas de potencial zoonótico, bem como restringir os agravos à sanidade dos gatos.

Nas amostras analisadas, obteve-se uma ocorrência de 75,6\% (68/90) para os helmintos e de 42,2\% (38/90) para os protozoários (Tabela 1). A maior ocorrência de helmintos nas fezes dos animais pode estar associada à ausência ou períodos prolongados entre tratamentos antiparasitários. MCGLADE et al. (2003) relatam que tratamentos realizados anualmente em gatos domésticos, diminuem em 0,2 vezes a possibilidade de parasitismo. Apesar da alta ocorrência de parasitas gastrointestinais nos gatos 
analisados, ao exame clínico, observou-se que todos os animais não apresentaram alterações tanto nos sinais vitais quanto nas condições corporais.

Ao analisar os diferentes fatores de risco que interferem na infecção de parasitos em gatos domésticos urbanos, observou-se que a escolaridade dos proprietários $(\mathrm{P}=0,0043)$ e a frequência de realização de tratamentos antiparasitários $(\mathrm{P}=0,0001)$ foram as variáveis que exerceram influências significativas sobre a ocorrência dos parasitos gastrointestinais. Verificou-se que o risco de parasitismo pode ser influenciado pelo nível de escolaridade do proprietário, assim sendo as chances de parasitismo aumentam em 6,8 vezes $(\mathrm{OR}=6,892$;
$\mathrm{P}=0,007)$ em animais de proprietários que possuem somente o ensino fundamental; enquanto que o risco de parasitismo diminui em 0,4 vezes $(\mathrm{OR}=0,417$; $\mathrm{P}=0,007)$ em animais de proprietários com ensino superior (Tabela 2). Observou-se também que animais não tratados com antiparasitários apresentam 4,8 vezes $(\mathrm{OR}=4,840 ; \mathrm{P}<0,001)$ mais chances de adquirir parasitas. Os fatores de risco identificados por MIRCEAN et al. (2010) diferiram deste estudo, no qual foi constatado que a idade e o meio ambiente de criação dos animais (urbano ou rural) foram os fatores que exerceram influências positivas no parasitismo. CAPÁRI et al. (2013) constataram que o uso de tratamentos antiparasitários diminuiu a frequência de

Tabela 2 - Fatores de risco associados à presença de parasitas gastrointestinais em gatos urbanos domésticos, avaliados no período de março a junho de 2011, na cidade de Santa Maria, RS.

\begin{tabular}{|c|c|c|c|c|c|}
\hline \multirow{2}{*}{ Fator } & \multirow{2}{*}{ Categoria } & \multicolumn{2}{|c|}{----Parasitas Gastrointestinais---- } & \multirow{2}{*}{$\mathrm{X}^{2}$ (Valor $\left.\mathrm{P}\right)$} & \multirow{2}{*}{ OR (Valor P) } \\
\hline & & $\operatorname{Sim}(n)$ & Não (n) & & \\
\hline \multirow{2}{*}{ Raça } & SRD & 82 & 91 & \multirow{2}{*}{$0,0571(0,8111)$} & $1,126(1,000)$ \\
\hline & Raça Pura & 8 & 10 & & $0,887(1,000)$ \\
\hline \multirow{2}{*}{ Sexo } & Macho & 40 & 41 & \multirow{2}{*}{$0,2889(0,5909)$} & $1,171(0,660)$ \\
\hline & Fêmea & 50 & 60 & & $0,854(0,660)$ \\
\hline \multirow{5}{*}{ Idade } & Até 60 dias & 4 & 3 & \multirow{5}{*}{$5,0929(0,2779)$} & $1,519(0,708)$ \\
\hline & De 2 a 6 meses & 15 & 10 & & $1,820(0,199)$ \\
\hline & 6 meses a 1 ano & 17 & 23 & & $0,789(0,594)$ \\
\hline & 1 ano a 5 anos & 43 & 43 & & $1,234(0,560)$ \\
\hline & + de 5 anos & 11 & 22 & & $0,500(0,088)$ \\
\hline \multirow{2}{*}{ Alimentação } & Ração & 72 & 83 & \multirow{3}{*}{$0,1476(0,7008)$} & $0,867(0,715)$ \\
\hline & Comida caseira & 18 & 18 & & $1,153(0,715)$ \\
\hline \multirow{3}{*}{ Alojamento do animal } & Casa & 57 & 62 & & $1,087(0,881)$ \\
\hline & Apartamento & 24 & 30 & \multirow[t]{2}{*}{$0,2441(0,8851)$} & $0,860(0,747)$ \\
\hline & Centro de criação & 9 & 9 & & $1,136(0,809)$ \\
\hline \multirow{2}{*}{ Acesso a área pública } & $\operatorname{Sim}$ & 61 & 59 & \multirow{2}{*}{$1,7860(0,1814)$} & $1,497(0,230)$ \\
\hline & Não & 29 & 42 & & $0,667(0,230)$ \\
\hline \multirow{5}{*}{$\begin{array}{l}\text { Frequência de tratamento } \\
\text { antiparasitário }\end{array}$} & 1/ano & 2 & 0 & \multirow{5}{*}{$28,7549(0,0001)$} & $5,734(0,220)$ \\
\hline & 2/ano & 11 & 20 & & $0,563(0,173)$ \\
\hline & 3/ano & 23 & 47 & & $0,394(0,002)$ \\
\hline & +3/ano & 5 & 14 & & $0,365(0,088)$ \\
\hline & Não tratado & 49 & 20 & & $4,840(<0,001)$ \\
\hline \multirow{2}{*}{ Meio ambiente higienizado } & Sim & 82 & 88 & \multirow{2}{*}{$0,7713(0,3798)$} & $1,514(0,488)$ \\
\hline & Não & 8 & 13 & & $0,660(0,488)$ \\
\hline \multirow{3}{*}{ Escolaridade do proprietário } & $\begin{array}{l}\text { Ensino } \\
\text { fundamental }\end{array}$ & 11 & 2 & \multirow{3}{*}{$10,8882(0,0043)$} & $6,892(0,007)$ \\
\hline & Ensino médio & 25 & 20 & & $1,558(0,232)$ \\
\hline & Ensino superior & 54 & 79 & & $0,417(0,007)$ \\
\hline
\end{tabular}


parasitismo em gatos domésticos na Hungria, estando de acordo com os dados encontrados neste trabalho. BAJER et al. (2011), em estudo realizado em cães, demonstraram que animais tratados sistematicamente com antiparasitários, mantiveram-se isentos de parasitas e em bom estado de saúde. Além disso, houve diminuição do fator de risco de transmissão de agentes parasitários, o que também foi concordante com esta pesquisa.

Em um estudo realizado por STALLIVIERE et al. (2009), na cidade de Lages, $\mathrm{SC}$, foi verificada maior prevalência de helmintos intestinais em felinos urbanos, cujos proprietários habitavam a área periférica e possuíam baixo poder aquisitivo. Esses fatores podem estar influenciando na oferta de boas condições sanitárias aos animais.

Foi constatado que tanto a raça $(\mathrm{P}=0,8111)$ quanto o sexo $(\mathrm{P}=0,5909)$ dos animais não exerceram influência sobre a ocorrência dos parasitas nos animais avaliados (Tabela 2). Esses dados estão de acordo com os estudos conduzidos por SERRA et al. (2003) e FUNADA et al. (2007). O sexo dos felinos $(\mathrm{P}=0,5909)$ não foi um fator de risco significativo neste trabalho. Observa-se que os gatos machos não castrados apresentam, como características da espécie, um maior deslocamento em busca de fêmeas para o acasalamento e demarcação territorial, podendo estar mais predispostos à exposição de formas parasitárias infectantes no meio ambiente. Entretanto, não se pode afirmar esse fato a animais castrados e confinados em apartamentos, o qual não foi considerado neste estudo. A idade dos animais parasitados não influenciou na porcentagem de positividade $(\mathrm{P}=0,277)$ (Tabela 2$)$, assim como foi constatado no México por MARTINEZBARBABOSA et al. (2003). OGASSAWARA et al. (1986) observaram percentuais maiores de $\boldsymbol{T}$. cati em gatos, principalmente até os seis meses de idade $(35,8 \%)$, e atribuíram essa condição à infecção galactogênica, logo após o nascimento. FUNADA et al. (2007) e CAPÁRI et al. (2013) demonstraram uma correlação positiva de parasitismo com gatos mais jovens, demonstrando a necessidade de tratamentos antiparasitários a serem administrados nessa categoria animal. Cabe ressaltar que alguns parasitas são mais frequentes em animais jovens, como, por exemplo, T. cati, sugerindo que animais dessa categoria são mais susceptíveis à infecção e algumas formas de resistência às reinfecções são desenvolvidas nos animais adultos (BALASSIANO et al., 2009).

Notou-se que grande parte dos animais (155/191) recebeu ração comercial em detrimento da alimentação caseira (36/191) (Tabela 2), o que indica haver um melhor status nutricional e, por conseguinte, uma eficiente capacidade de resposta imunológica aos parasitas por esses animais. Diferentemente de BALASSIANO et al. (2009), no presente estudo, não foi possível estabelecer uma correlação positiva entre o parasitismo e o tipo de alimentação ofertada aos felinos $(\mathrm{P}=0,7008)$.

$\mathrm{O}$ alojamento dos gatos $(\mathrm{P}=0,8851)$ e o acesso às áreas públicas $(\mathrm{P}=0,1814)$ (Tabela 2) também não influenciaram na ocorrência dos parasitas gastrointestinais encontrada neste estudo. No entanto, MIRCEAN et al. (2010) constataram que o acesso dos animais às áreas públicas proporcionou um maior contato do hospedeiro com as formas infectantes existentes no meio ambiente. BALASSIANO et al. (2009), quando avaliaram essa variável, não obtiveram diferenças de prevalência de parasitas em cães; porém, ressaltaram uma maior possibilidade dos animais que têm acesso ao solo estar mais exposto às formas parasitárias infectantes. Essa fonte de infecção pode estar relacionada com a ausência de condições mínimas de higiene da localidade, o que pode proporcionar um aumento no risco de infecção e/ ou pode exacerbar a translação parasitária ao animal. Esses autores relataram a importância de realizar medidas estratégicas de controle de parasitos em felídeos para reduzir a contaminação ambiental. Neste trabalho, não foi observada a associação de higiene e remoção de fezes $(\mathrm{P}=0,3798)$ e infecção parasitária, o que também foi constatado por BALASSIANO et al. (2009). A remoção de fezes reduz a contaminação de formas infectantes existentes no meio ambiente e pode ocasionar a redução do risco de infecção do homem e dos animais.

\section{CONCLUSÃO}

A ocorrência de endoparasitos nos gatos urbanos de Santa Maria-RS, avaliada neste estudo, é relativamente alta, sendo mais frequente a presença de um agente com potencial zoonótico, Toxocara spp., em mono e multi-infecções. Os fatores de risco relacionados à infecção de parasitas gastrointestinais identificados neste trabalho foram, sobretudo, a escolaridade dos proprietários e a frequência de tratamentos antiparasitários realizados nos animais. Os resultados obtidos nesta pesquisa contribuem para o conhecimento da epidemiologia das parasitoses gastrointestinais dos gatos domiciliados no município estudado. Além disso, esses dados poderão auxiliar médicos veterinários e proprietários na elaboração de estratégias de controle e profilaxia antiparasitária para gatos domésticos. 


\section{COMITÊ DE ÉTICA E BIOSSEGURANÇA}

O projeto foi avaliado pelo Comitê de Ética em Pesquisa com Seres Humanos (CEP)/UFSM, e aprovado no sistema CEP/Conep sob CAAE n. 05982412.0.0000.5346.

\section{REFERENCIAS}

ALMEIDA, M.; M. AYRES. Considerações gerais sobre os antihelmínticos. In: SPINOSA, H.S. et al. Farmacologia aplicada à medicina veterinária. 4 ed. Rio de Janeiro: Guanabara Koogan, 2006. v.4, p.459-466.

BAJER, A. et al. Risk factors and control of intestinal parasite infections in sled dogs in Poland. Veterinary Parasitology, v.175, n.3-4, p.343-350, 2011. Disponível em: <http://www.ncbi. nlm.nih.gov/pubmed/21075531>. Acesso em: 23 nov. 2011. doi: 10.1016/j.vetpar.2010.10.029

BALASSIANO, B.C. et al. Factors associated with gastrointestinal parasite infection in dogs in Rio de Janeiro, Brazil. Preventive Veterinary Medicine, v.91, n.2-4, p.234-240, 2009. Disponível em: <http://www.ncbi.nlm.nih.gov/pubmed/19577316>. Acesso em: 23 nov. 2011. doi: 10.1016/j.prevetmed.2009.05.030.

CAPÁRI, B. et al. Parasitic infections of domestic cats, Felis catus, in western Hungary. Veterinary Parasitology, v.192, p.3342, 2013. Disponível em: <http://www.sciencedirect.com/science/ article/pii/S0304401712005948>. Acesso em: 16 nov. 2013. doi: 10.1016/j.vetpar.2012.11.011

COELHO, W.M.D. et al. Ocorrência de parasitos gastrintestinais em amostras fecais de felinos no município de Andradina, São Paulo. Revista Brasileira de Parasitologia Veterinária, v.18, n.2, p.46-49, 2009. Disponível em: <http://www.scielo.br/pdf/ rbpv/v18n2/a10v18n2.pdf $>$. Acesso em: 23 nov. 2011. doi: $10.4322 /$ rbpv.01802010.

DALL'AGNOL, L.P. et al. Parasitos gastrintestinais em gatos naturalmente infectados no município de Santa Maria no estado do Rio Grande do Sul, Brasil. Acta Veterinaria Brasilica, v.4, n.3, p.181-184, 2010. Disponível em:<http://periodicos.ufersa.edu.br/ revistas/index.php/acta/article/view/1781/4678>. Acesso em: 23 nov. 2011.

FUNADA, M. et al. Frequency of gastrointestinal parasites in dogs and cats referred to a veterinary school hospital in the city of São Paulo. Arquivo Brasileiro de Medicina Veterinária e Zootecnia, v.59, n.5, p.1338-1340, 2007. Disponível em: <http:// www.scielo.br/pdf/abmvz/v59n5/a38v59n5.pdf>. Acesso em: 23 nov. 2011.

LORENZINI, G. et al. Prevalence of intestinal parasites in dogs and cats under veterinary care in Porto Alegre, Rio Grande do
Sul, Brazil. Brazilian Journal of Veterinary Research Animal Science, v.44, n.2, p.137-145, 2007. Disponível em: <http://www. revistasusp.sibi.usp.br/pdf/bjvras/v44n2/10.pdf >. Acesso em: 23 nov. 2011

MARTINEZ-BARBABOSA, I. et al. The prevalence of Toxocara cati in domestic cats in Mexico City. Veterinary Parasitology, v.114, n.1, p.43-49, 2003. Disponível em: <http://www.ncbi.nlm. nih.gov/pubmed/12732465>. Acesso em: 23 nov. 2011.

McGLADE, T.R. et al. Gastrointestinal parasites of domestic cats in Perth, Western Australia. Veterinary Parasitology, v.117, n.4, p.251-262, 2003. Disponível em: <http://www.ncbi.nlm.nih.gov/ pubmed/14637027>. Acesso em: 23 nov. 2011.

MIRCEAN, V. et al. Prevalence of endoparasites in household cat (Felis catus) populations from Transylvania (Romania) and association with risk factors. Veterinary Parasitology, v.171, n.1-2, p.163-166, 2010. Disponível em: <http://www.ncbi.nlm. nih.gov/pubmed/20381250>. Acesso em: 23 nov. 2011. doi: 10.1016/j.vetpar.2010.03.005.

MONTEIRO, S.V. Técnicas laboratoriais. In: MONTEIRO, S.V. Parasitologia na Medicina Veterinária. São Paulo: Roca, 2010. Cap.29, p.301-312.

OGASSAWARA, S. et al. Prevalence of internal parasitisms in cats in city of São Paulo, Brazil. Revista da Faculdade de Medicina Veterinária e Zootecnia da Universidade de São Paulo, v.23, n.1, p.39-46, 1986.

PALMER, C.S. et al. National study of the gastrointestinal parasites of dogs and cats in Australia. Veterinary Parasitology, v.151, n.2-4, p.181-190, 2008. Disponível em: <http://www.ncbi. nlm.nih.gov/pubmed/18055119>. Acesso em: 23 nov. 2011.

SERRA, C.M.B. et al. Exame parasitológico de fezes de gatos (Felis catus domesticus) domiciliados e errantes da Região Metropolitana do Rio de Janeiro, Brasil. Revista da Sociedade Brasileira de Medicina Tropical, v.36, n.3, p.331-334, 2003. Disponível em: <http://www.scielo.br/scielo.php?pid=S0037$86822003000300003 \&$ script=sci_abstract\&tlng=pt>. Acesso em: 23 nov. 2011.

STALLIVIERE, F.M. et al. Ectoparasites and intestinal helminths in Felis catus domesticus from Lages city, SC, Brazil and social-economical and cultural aspects of owners of family pets. Revista Brasileira de Parasitologia Veterinária, v.18, n.4, p.26-31, 2009. Disponível em: <http://www.cbpv.com.br/rbpv/ documentos/1842009/rbpv.01804005.pdf>. Acesso em: 23 nov. 2011. doi: $10.4322 /$ rbpv.01804005.

THURSFIELD, M.V. Epidemiologia veterinária. 2.ed. São Paulo: Roca, 2004. 556p. 